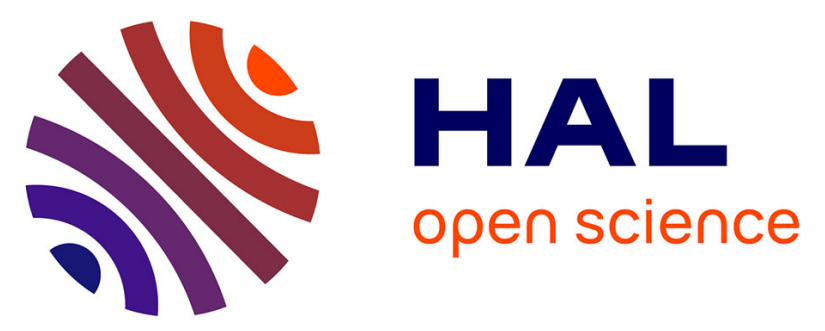

\title{
Concept of Lattice Mismatch and Emergence of Surface States in Two-dimensional Hybrid Perovskite Quantum Wells
}

Mikael Kepenekian, Boubacar Traore, Jean-Christophe Blancon, Laurent Pedesseau, Hsinhan Tsai, Wanyi Nie, Constantinos C Stoumpos, Mercouri G Kanatzidis, Jacky Even, Aditya D Mohite, et al.

\section{To cite this version:}

Mikael Kepenekian, Boubacar Traore, Jean-Christophe Blancon, Laurent Pedesseau, Hsinhan Tsai, et al.. Concept of Lattice Mismatch and Emergence of Surface States in Two-dimensional Hybrid Perovskite Quantum Wells. Nano Letters, 2018, 18 (9), pp.5603-5609. 10.1021/acs.nanolett.8b02078 . hal-01859530

\author{
HAL Id: hal-01859530 \\ https://hal.science/hal-01859530
}

Submitted on 18 Oct 2018

HAL is a multi-disciplinary open access archive for the deposit and dissemination of scientific research documents, whether they are published or not. The documents may come from teaching and research institutions in France or abroad, or from public or private research centers.
L'archive ouverte pluridisciplinaire HAL, est destinée au dépôt et à la diffusion de documents scientifiques de niveau recherche, publiés ou non, émanant des établissements d'enseignement et de recherche français ou étrangers, des laboratoires publics ou privés. 


\section{Concept of lattice mismatch and emergence of surface states in 2D hybrid perovskite quantum wells}

Mikaël Kepenekian, ${ }^{* \dagger}$ Boubacar Traore ${ }^{\dagger}$ Jean-Christophe Blancon,+ Laurent Pedesseau, "Hsinhan Tsai, ${ }^{\ddagger}$ Wanyi Nie, $\stackrel{\ddagger}{\ddagger}$ Constantinos C. Stoumpos,,$\|$ Mercouri

G. Kanatzidis," Jacky Even, " Aditya D. Mohite,,$^{\ddagger}$ Sergei Tretiak,,$+\ddagger$ and Claudine Katan ${ }^{*, \dagger}$

$\dagger$ Univ Rennes, ENSCR, INSA Rennes, CNRS, ISCR - UMR 6226, F-35000 Rennes, France

$\ddagger$ Los Alamos National Laboratory, Los Alamos, NM 87545, U.S.A.

\Univ Rennes, INSA Rennes, CNRS, FOTON - UMR 6082, F-35000 Rennes, France $\S$ Department of Materials Science and Nanoengineering, Rice University, Houston, TX 77005, USA

|| Department of Chemistry, Northwestern University, Evanston, IL 60208, USA

E-mail: mikael.kepenekian@univ-rennes1.fr; serg@lanl.gov; claudine.katan@univ-rennes1.fr 


\begin{abstract}
Surface states are ubiquitous to semiconductors and significantly impact the physical properties and consequently the performance of optoelectronic devices. Moreover, surface effects are strongly amplified in lower dimensional systems such as quantum wells and nanostructures. Layered halide perovskites (LHPs) are 2D solution-processed natural quantum wells where optoelectronic properties can be tuned by varying the perovskite layer thickness $n$, i.e. the number of octahedra spanning the layer. They are efficient semiconductors with technologically relevant stability. Here, a generic elastic model and electronic structure modelling are applied to LHPs heterostructures with various layer thickness. We show that the relaxation of the interface strain is triggered by perovskite layers above a critical thickness. This leads to the release of the mechanical energy arising from the lattice mismatch, which nucleates the surface reorganization and may potentially induce the formation of previously observed lower energy edge states. These states, which are absent in 3D perovskites are anticipated to play a crucial role in the design of LHPs for optoelectronic systems.
\end{abstract}

\title{
Keywords
}

halide perovskites, layered materials, lattice mismatch, surface states, exciton, density functional theory

\section{Table of Contents Graphical Abstract}

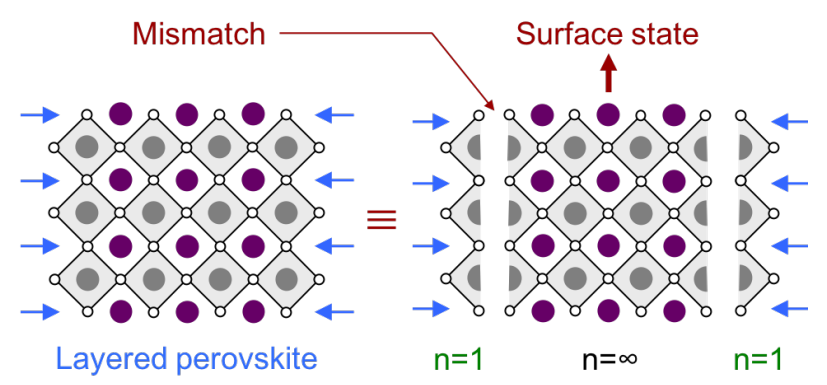


Surfaces and interfaces are known to play a central part in the performances of classical semiconductor based devices. ${ }^{1-3}$ This holds true for the recently emerged halide perovskites. ${ }^{4,5}$ The 2D members of the family, layered halide perovskites (LHPs) are 2D solutionprocessed natural quantum wells, ${ }^{6-8}$ and present superior photo- and chemo- stability compared to their 3D counterparts. They show strong promise in high performance optoelectronic devices such as photovoltaics, field effect transistors, electrically injected light emission and polarized optical spin injection. ${ }^{9-14}$ Their properties depend on the number $\mathrm{n}$ of $\mathrm{MX}_{6}$ octahedra that span the perovskite layer ( $\mathrm{M}$ is a metal, $\mathrm{X}$ a halogen). As in classical semiconductors ${ }^{2}$ surface and interface structures can have a strong influence on the properties of LHPs. ${ }^{15}$ While experimental results exists, especially in Ruddlesden-Popper perovskites (RPPs) of general formula $\mathrm{A}_{2}^{\prime} \mathrm{A}_{\mathrm{n}-1} \mathrm{M}_{\mathrm{n}} \mathrm{X}_{3 \mathrm{n}+1}$ ( $\mathrm{A}$ and $\mathrm{A}^{\prime}$ being cations), there is no simple model to predict and control LHP surface properties.

Here, we design a model based on the concept of lattice mismatch, considering LHPs as heterostructures built from the $\mathrm{n}=1$ monolayered perovskite $\mathrm{A}_{2}{ }_{2} \mathrm{MX}_{4}$ and the $\mathrm{n}=\infty 3 \mathrm{D}$ $\mathrm{AMX}_{3}$. The capacities of the model are first examined taking the RPPs of general formula $(\mathrm{BA})_{2}(\mathrm{MA})_{\mathrm{n}-1} \mathrm{~Pb}_{\mathrm{n}} \mathrm{I}_{3 \mathrm{n}+1}$. This specific family of RPPs is further inspected by means of DFT atomistic calculations, showing that their optical properties are decisively impacted by surface relaxations occurring for structures with $n>2$. The general picture that emerges from the combined study leads to understanding of physical phenomena underpinning surface reconstruction and concomitant modifications of electronic structure, and allows to formulate the design principles of LHP materials optimized for optoelectronics, solid-state lighting or photovoltaics.

It has been shown earlier that ordered LHPs structures can be treated as composite L1/L2 systems with coherent interfaces, although the definition of the L1 and L2 bulk materials must be chosen with care. ${ }^{16}$ Such a framework allows relevant analysis of electronic and dielectric properties of the composite. ${ }^{16,17}$ To investigate mechanical properties, we construct a model (see Text I, Supporting Information (SI) for details) based on the theory 

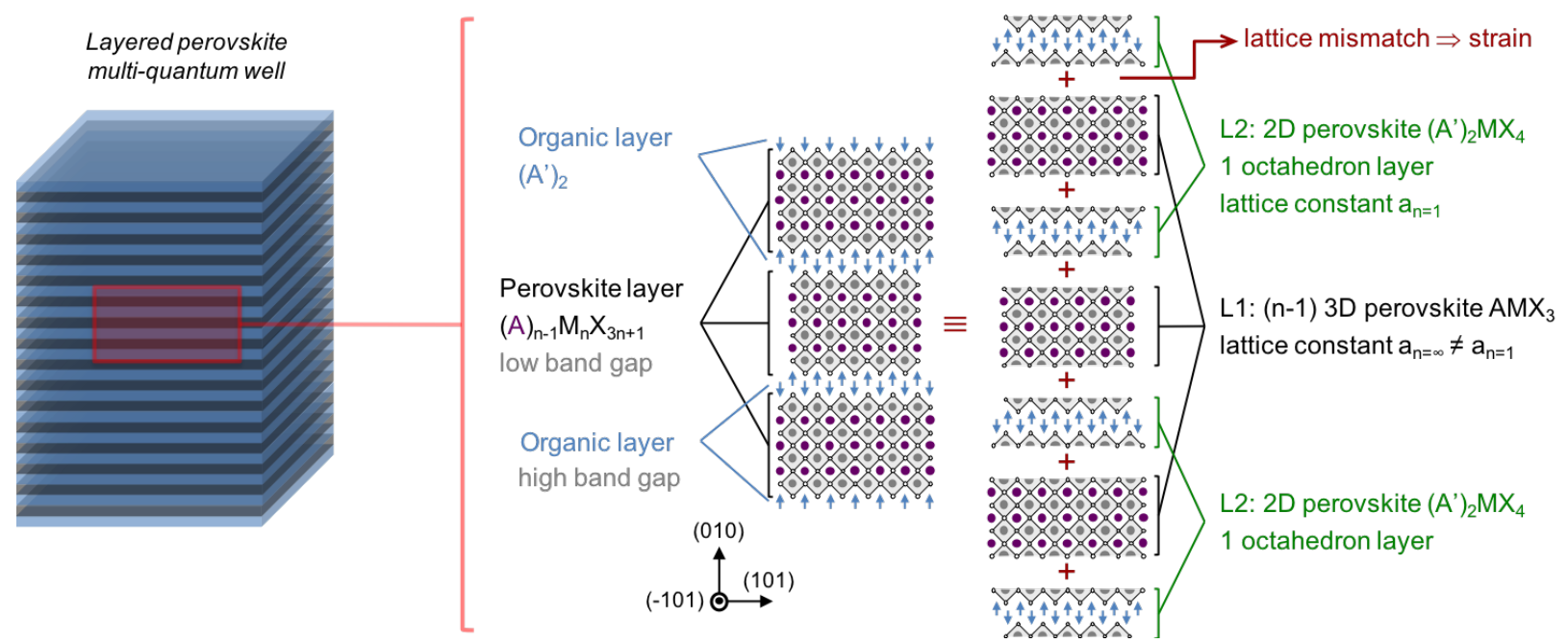

Figure 1: Schematics of hybrid layered compounds regarded as heterostructures L1/L2 with L1 the $3 \mathrm{D}(\mathrm{n}=\infty)$ bulk material, e.g. $\mathrm{MAPbI}_{3}$, and $\mathrm{L} 2$, a $\mathrm{n}=1$ compound, e.g. $(\mathrm{BA})_{2} \mathrm{PbI}_{4}$.

of elasticity in classical semiconductor heterostructures ${ }^{18}$ by identifying the LHP structure with a multi-quantum well system (Fig. 1) with alternating stacking of 3D perovskite layers L1 $\left(\mathrm{AMX}_{3}\right.$, of thickness n-1) and of 2D perovskite monolayers L2 (single octahedron, $\left.\mathrm{n}=1\right)$. This combination forms an interface between two structurally-different layers, equivalent to a so-called L1/L2 heterostructure with a coherent interface (lattices are continuous across the interface in two directions). ${ }^{19}$

A first simple approach may be inspired by the elastic model used to predict the influence of lattice mismatch during epitaxial growth of conventional semiconductor heterostructures having a coherent interface. ${ }^{18,19}$ For an epitaxial layer with a low lattice mismatch $\left(\epsilon_{11}=\epsilon_{22}\right)$ and the condition for a free $(001)$ surface $\left(\sigma_{33}=0\right)$ on a rigid (001) zinc-blende substrate, the relation between stress and strain tensors (with $C_{i j}$ the components of the tensor) leads to the out-of-plane strain $\epsilon_{33}=\left(-2 C_{12} \epsilon_{11}\right) / C_{11}$. Alternatively, the same result can be obtained by minimizing the elastic free energy with respect to $\epsilon_{33}$. This model must be adapted for LHPs. The elastic properties of both L1 and L2 layers must be taken into account with $\epsilon_{i j}$ transforming to $\epsilon_{i j, L 1}$ and $\epsilon_{i j, L 2}$ and $C_{i j}$ to $C_{i j, L 1}$ and $C_{i j, L 2}$ (Fig. 2a). Considering the transverse elastic approximation for $\mathrm{L} 1=(\mathrm{n}-1) \mathrm{AMX}_{3}$, then, the total elastic energy of the 
L1/L2 composite heterostructure is given by:

$$
\begin{aligned}
F & =\frac{1}{2} S l_{L 1}\left[C_{11, L 1}\left(\epsilon_{11, L 1}^{2}+\epsilon_{22, L 1}^{2}+\epsilon_{33, L 1}^{2}\right)\right. \\
& \left.+2 C_{12, L 1}\left(\epsilon_{11, L 1} \epsilon_{22, L 1}+\epsilon_{11, L 1} \epsilon_{33, L 1}+\epsilon_{22, L 1} \epsilon_{33, L 1}\right)\right] \\
& +\frac{1}{2} S l_{L 2}\left[C_{11, L 2}\left(\epsilon_{11, L 2}^{2}+\epsilon_{22, L 2}^{2}\right)+C_{33, L 2} \epsilon_{33, L 2}^{2}+2 C_{12, L 2} \epsilon_{11, L 2} \epsilon_{22, L 2}\right. \\
& \left.+2 C_{13, L 2}\left(\epsilon_{11, L 2} \epsilon_{33, L 2}+\epsilon_{22, L 2} \epsilon_{33, L 2}\right)\right]
\end{aligned}
$$

where $S$ is the surface of the coherent interface, $l_{L 1}=(n-1) c_{3 D}$ and $l_{L 2}=c_{2 D}$ are the thicknesses of the two layers, $c_{2 D}$ and $c_{3 D}$ are the stacking parameters of $\left(\mathrm{A}^{\prime}\right)_{2} \mathrm{MX}_{4}$ and $\mathrm{AMX}_{3}$, respectively. Considering that the two layers share the same in-plane lattice parameter $a$ and the conditions for free strain relaxation along the stacking axis, the equilibrium in-plane lattice parameter $a_{e q}$ is derived by minimizing the total energy, yielding:

$$
a_{e q}=\frac{\frac{K_{L 1}}{a_{3 D}}+\frac{K_{L 2}}{a_{2 D}}}{\frac{K_{L 1}}{a_{3 D}^{2}}+\frac{K_{L 2}}{a_{2 D}^{2}}}
$$

$$
\begin{array}{r}
\text { with } K_{L 1}=(n-1) c_{3 D}\left(C_{11, L 1}+C_{12, L 1}-2 \frac{C_{12, L 1}^{2}}{C_{11, L 1}}\right) \\
\text { and } K_{L 2}=c_{2 D}\left(C_{11, L 2}+C_{12, L 2}-2 \frac{C_{13, L 2}^{2}}{C_{33, L 2}}\right)
\end{array}
$$

To illustrate this general concept, we consider the family of RPPs of general formula $(\mathrm{BA})_{2}(\mathrm{MA})_{\mathrm{n}-1} \mathrm{~Pb}_{\mathrm{n}} \mathrm{I}_{3 \mathrm{n}+1}$ that can be synthesized in phase-pure form (only one n-value). ${ }^{20-22}$ It can be approximated to an L1/L2 heterostructure with $\mathrm{L} 1=(\mathrm{n}-1) \mathrm{MAPbI}_{3}$ and $\mathrm{L} 2=(\mathrm{BA})_{2} \mathrm{PbI}_{4}$ (Fig. 1). Fig. 2b represents experimentally observed variations of the in-plane average lattice parameter (perpendicular to the stacking axis of the LHP, i.e. to (010)) as a function of $n$ for the native $(\mathrm{BA})_{2}(\mathrm{MA})_{\mathrm{n}-1} \mathrm{~Pb}_{\mathrm{n}} \mathrm{I}_{3 \mathrm{n}+1}$ heterostructure, as well as the out-of plane lattice parameters (along the stacking axis of the LHP) for the end members of the homologous 

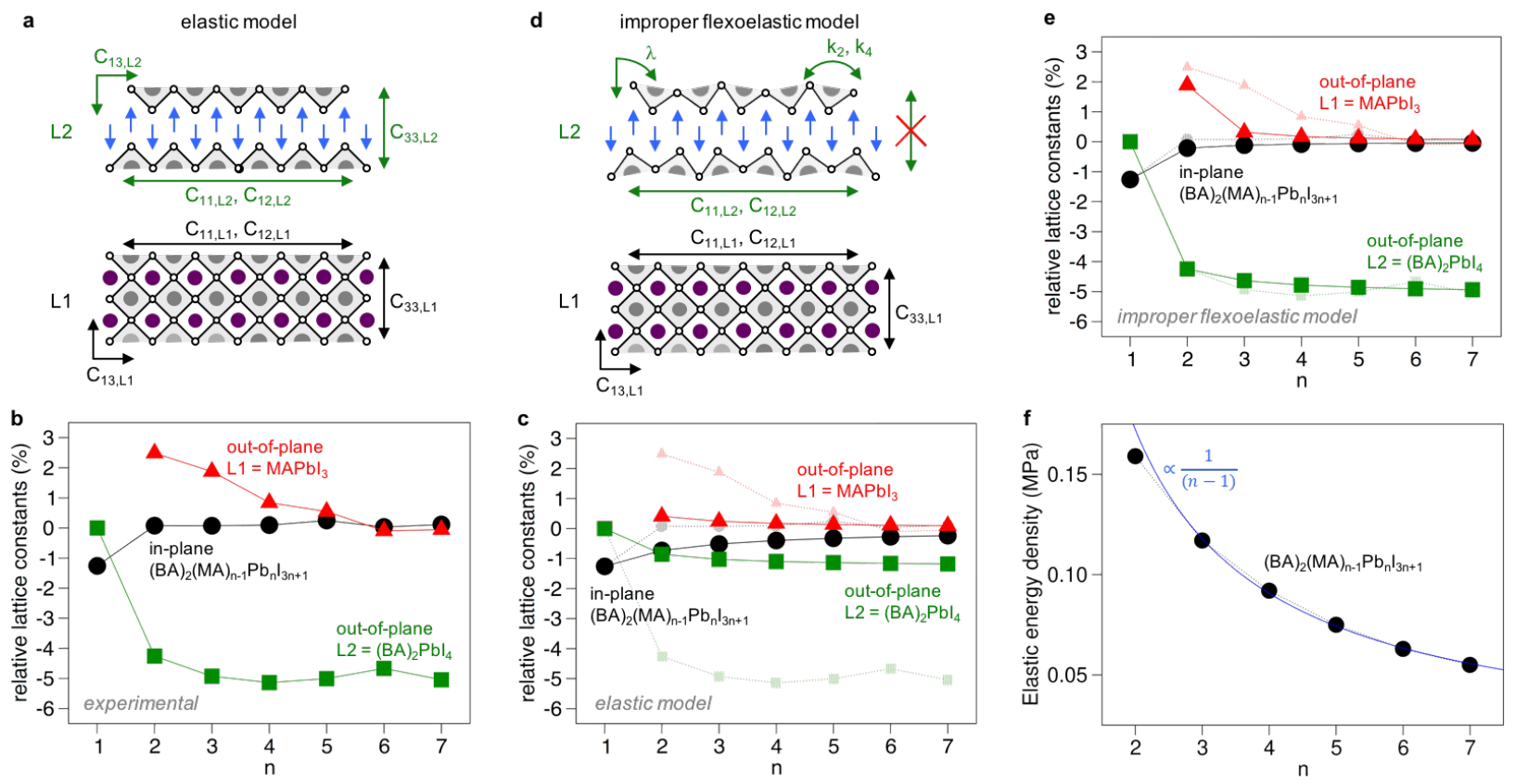

Figure 2: (a) Parameters of the purely elastic model, relying on the strain tensor $\left\{C_{i j}\right\}$. (b) In-plane expansion and out-of-plane contractions of experimental lattice constants for $(\mathrm{BA})_{2}(\mathrm{MA})_{\mathrm{n}-1} \mathrm{~Pb}_{n} \mathrm{I}_{3 \mathrm{n}+1}$ and the L1 and L2 layers. The room-temperature structures of $\mathrm{MAPbI}_{3}$ and $(\mathrm{BA})_{2} \mathrm{PbI}_{4}$ serve as references for L1 and L2 structures, respectively. (c) Same from the purely elastic model. (d) Parameters of the improper flexoelastic model. L2 now react to the strain by also modifying the in-plane and out-of-plane tilting angles through $\lambda, k_{2}$ and $k_{4}$ (see text and Text I, SI). (e) Same as (b) from the improper flexoelastic model (see SI for details). (f) Computed elastic energy density for the $(\mathrm{BA})_{2}(\mathrm{MA})_{\mathrm{n}-1} \mathrm{~Pb}_{n} \mathrm{I}_{3 \mathrm{n}+1}$ heterostructure (black circles). Non-linear fit related to the ratio between the volume occupied by L2 (1) and L1 (n-1) layers in the L1/L2 heterostructure leading to a $1 /(\mathrm{n}-1)$ behavior.

series L1 ((n-1)MAPbI$\left.)_{3}\right)$ and L2 $\left(n=1,(B A)_{2} \mathrm{PbI}_{4}\right)$. As qualitatively predicted based on elasticity (Fig. 2c), the in-plane lattice expansion from $n=1$ to $n=\infty$, gives rise to an out-ofplane lattice contraction in both L1 and L2 layers. However, the experimental variation of the in-plane parameter is noticeably steep, the in-plane parameter of $\mathrm{MAPbI}_{3}(\mathrm{n}=\infty)$ being almost already recovered for $n=2$. A similar steep variation is observed for the out-of-plane lattice parameter of the L2 layer. The deficiency of a pure elastic model (Fig. 2c) can be traced back to additional rotational degrees of freedom, namely octahedral tilting, which also afford a path to relax the accumulated mechanical energy (Fig. 2d). In fact, quantitative agreement between experimental results (Fig. 2b) and model predictions (Fig. 2e) can only be obtained when considering that L1 reacts in a purely elastic manner, whereas L2 relaxes 
the strain through the variation of octahedra tilt angles (Fig. 2d). This can be observed on the angles extracted from the $(\mathrm{BA})_{2}(\mathrm{MA})_{\mathrm{n}-1} \mathrm{~Pb}_{\mathrm{n}} \mathrm{I}_{3 \mathrm{n}+1} \mathrm{RPP}$ experimental structures (see Text II, Fig. S1 and Table S1, SI). The octahedra tilt angles in L2 structure are indeed more important than in the L1 layers. In other words, the mechanical energy is more efficiently relaxed in the bulk RPPs structures by rotation of those octahedra that are directly in contact with the flexible organic cations, than by $\mathrm{Pb}-\mathrm{I}$ bond elongation.

Classic theory of elasticity predicts that, for a heterostructure L1/L2 with a large lattice mismatch between L1 and L2, the structure may undergo a reorganization for a critical layer thickness, to form nanostructures at the surface in order to relax the accumulated bulk mechanical energy. ${ }^{19}$ From the above results, the elastic energy density in RPPs with varying perovskite thickness $\mathrm{n}$ was computed (Fig. 2f). We observed a maximum elastic energy density of $\sim 0.16 \mathrm{MPa}$ for the $\mathrm{RPP} n=2$, and a monotonic reduction of this energy with increasing $\mathrm{n}$, which ultimately vanishes for bulk $3 \mathrm{D}$ perovskite $(\mathrm{n} \rightarrow \infty)$. The monotonic decrease of elastic energy density is related to the behavior of lattice constants. From Fig. 2f, one can see that starting from $n=3$, L1 is defining the geometry, whereas L2 carries the stress in the heterostructure. As $\mathrm{n}$ grows, the ratio between L2 and L1 volumes decreases concomitantly with the elastic energy density although lattice parameters remain constants. Therefore, elastic energy density arising from the interface is expected to have direct consequences over surface properties for RPPs with low n-values.

To gain microscopic insight of surface reorganization and their associate effect on physical properties, we further focus on the $(\mathrm{BA})_{2}(\mathrm{MA})_{\mathrm{n}-1} \mathrm{~Pb}_{\mathrm{n}} \mathrm{I}_{3 \mathrm{n}+1} \mathrm{RPP}(\mathrm{n}=1-4)$ using density functional theory (DFT). DFT allows modelling the structural relaxation at the relevant surfaces with account of all structural distortions (see Method, for computational details). Applications using RPPs as active materials mainly employ two different orientations; the 2D layers are either parallel or perpendicular to the substrate or an interface layer (Fig. 3a). The most relevant surface of the RPP is then the (101) surface (Fig. 3b), 9,15 which we model here on specifically designed slabs (Text III and Fig. S3, SI), labelled as bulk-like and sur- 

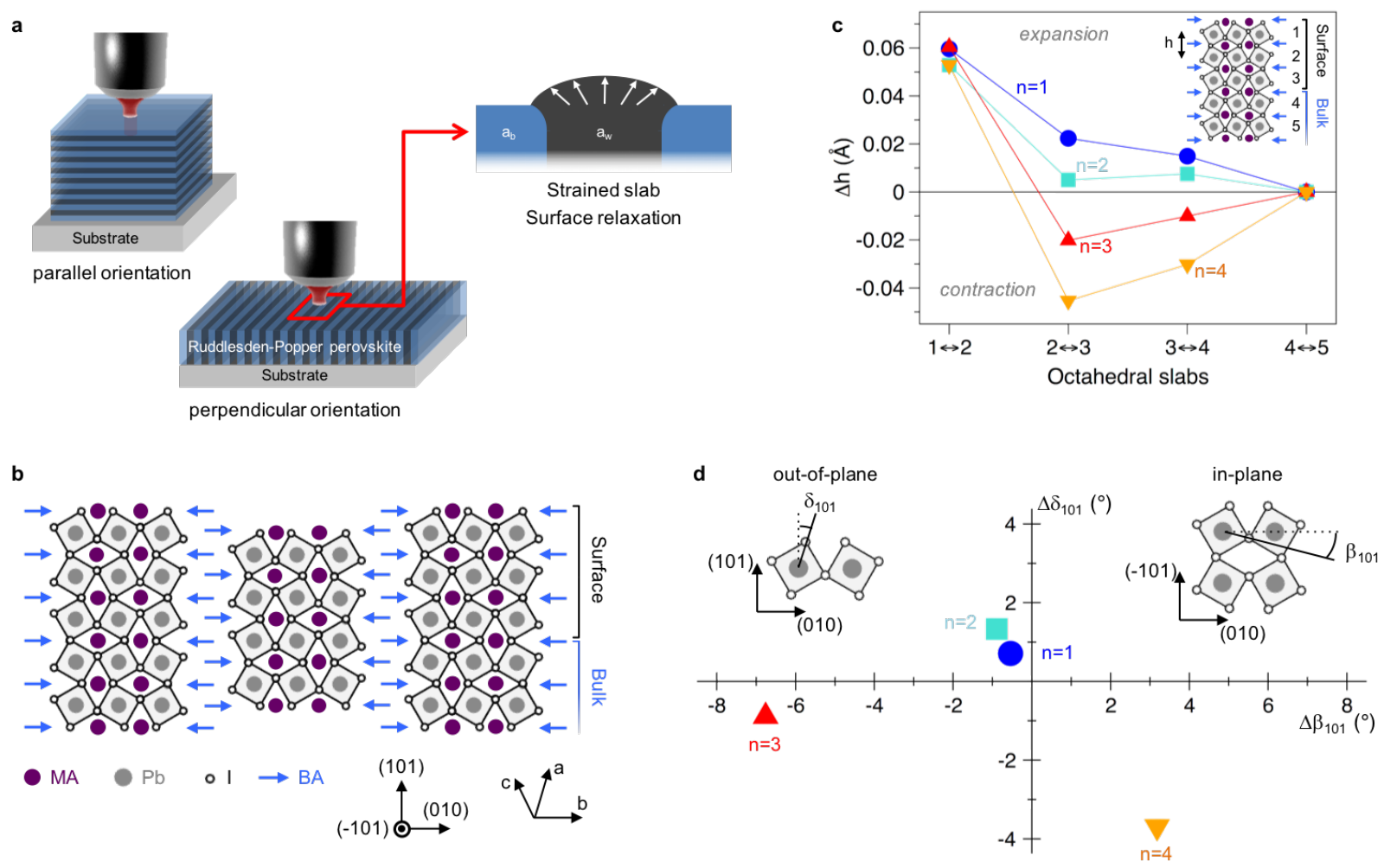

Figure 3: Surface relaxation in LHP multi-quantum wells. (a) Schematics of LHP-based devices in parallel and perpendicular orientation. (b) Schematics of the (101) surface of the layered perovskite $(\mathrm{BA})_{2}(\mathrm{MA})_{\mathrm{n}-1} \mathrm{~Pb}_{\mathrm{n}} \mathrm{I}_{3 \mathrm{n}+1}$ with $\mathrm{n}=3$. (c) Variation of the interlayer height difference $(\Delta \mathrm{h})$ from bulklike to surface (see inset). (d) Variation of in-plane $\left(\beta_{101}\right)$ and out-of-plane $\left(\delta_{101}\right)$ tiltings of surface octahedra due to the (101) surface relaxation.

face, for varying thickness $n=1$ to 4 . The calculated changes in the surface structure were represented by (i) the contraction/expansion of the octahedron slabs close to the surface in the (101) direction $\left(\Delta_{\mathrm{h}}\right.$, Fig. $\left.3 \mathrm{c}\right)$, and (ii) the in-plane $\left(\beta_{101}\right)$ and out-of-plane $\left(\delta_{101}\right)$ tilting of the octahedra close to the surface (Fig. 3d). ${ }^{7}$ This representation highlights our early conclusion drawn from the elastic model that rotational degree of freedom of the octahedra play an essential role in relaxing the internal elastic energy, in contrast with classical semiconductor descriptions where local strain tensor suffices. ${ }^{23}$ In an attempt to quantify the role of each degrees of freedom, we have built a model allowing us to enforce contraction or elongation of bonds independently from octahedron tiltings (Fig. S5, SI). Both mechanisms present similar contribution with a price of ca. $30 \mathrm{meV}$ for a $1 \%$ strain on the structure.

Fig. 3c shows the variation along the (101) direction of the distance $h$ between octahedral 
slabs close to the perovskite surface. The reference value of $\mathrm{h}$ is obtained from the bulklike region fixed in our DFT calculations $(4 \leftrightarrow 5$ distance in Fig. 3c). The evolution of the inter-slab distance yields two opposite behaviours for $n=1,2$ and $n>2$. For $n>2$, the surface slab expansion is accompanied by a contraction of the sub-surface slabs, which leads to a decoupling of the top surface octahedron slab from the sub-surface ones. On the other hand, for $\mathrm{n}=1,2$, expansion of octahedra slabs was observed in the entire surface region. A similar distinct behaviour between $n=1,2$ and $n>2$ was noted by analysing the surface relaxation in these BA-based RPPs occurring through in-plane and out-of-plane tilting of octahedra (Fig. 3d). In fact, surface octahedra in $n=1,2$ yield almost no rotational degree of freedom, whereas $n>2$ systems exhibit significantly larger tilting of surface octahedra. The drastic change of surface behavior, when increasing the perovskite layer thickness from $n=2$ to $n=3$ coincides with the appearance of specific signatures in the electronic structure of bulk RPP materials (Fig. S4, SI). In particular, the $\mathrm{Pb}-\mathrm{I}$ dangling bond located in the middle of the inorganic layer allows for a local strain relaxation (Fig. S6, SI). The occurrence of a central octahedron, whose surrounding resembles the one in $3 \mathrm{D}$ perovskite, appears essential to trigger structural relaxation of the internal elastic energy.

We evaluate the impact of these surface relaxation processes on the electronic and optical properties of RRPs by comparing band structures and wavefunctions at the surface and in the bulk (Fig. 4). The electronic band structure still presents a direct bandgap at the surface as compared to the bulk but with variation of the bandgap energy (Fig. 4a,b and Fig. S7, SI). We observe that the bandgap blueshifts by 70 and $150 \mathrm{meV}$ for $\mathrm{n}=1$ and 2 respectively and redshifts by 120 and $70 \mathrm{meV}$ for $\mathrm{n}=3$ and 4 , respectively. The accuracy of our approach is supported by (i) the excellent agreement between the calculated exciton properties in the bulk-like region with reported experimental results for the same materials (see detail in Text IV, SI), ${ }^{24}$ and (ii) the similar pattern in the optical bandgap shift between the RPP layer surface with respect to the bulk (Fig. S8, SI). ${ }^{15}$ According to surface relaxation results, lattice expansion at the (101) surface with relatively small octahedral tilting leads 

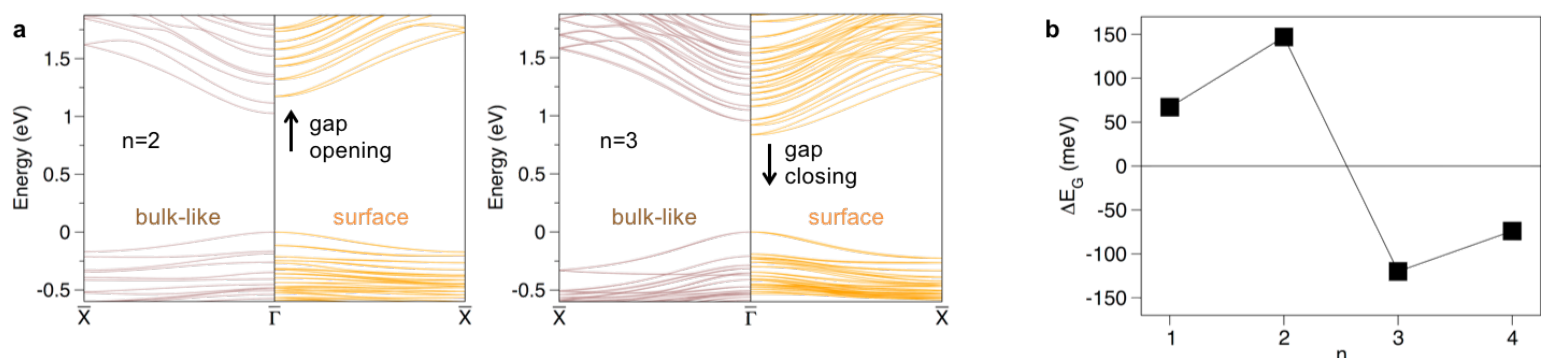

c
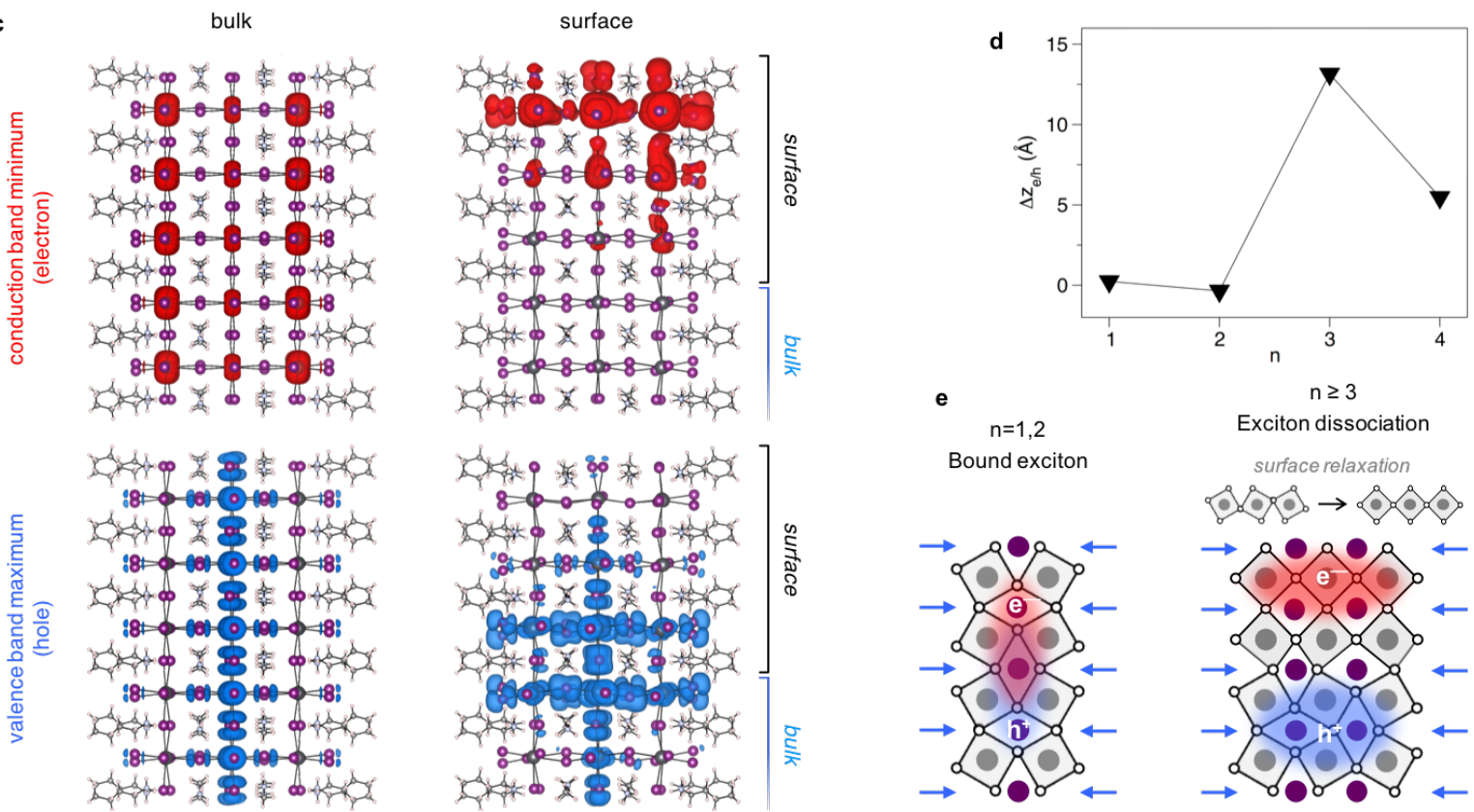

e

$\mathrm{n}=1,2$ Bound exciton

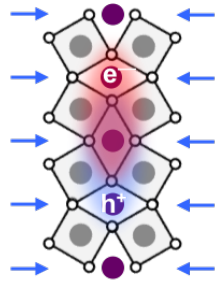

Exciton dissociation

surface relaxation $20 \% \rightarrow$

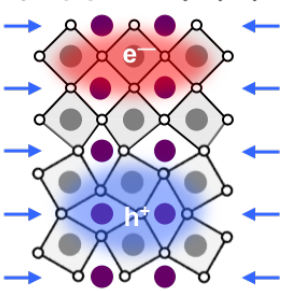

Figure 4: Impact of surface structural relaxation on electronic and optical properties in $(\mathrm{BA})_{2}(\mathrm{MA})_{\mathrm{n}-1} \mathrm{~Pb}_{\mathrm{n}} \mathrm{I}_{3 \mathrm{n}+1}$. (a) Slab band structures in the bulk-like (left) and relaxed (101) surface (right) for $\mathrm{n}=2$ and 3. (b) DFT variation of $\mathrm{E}_{\mathrm{G}}$ going from bulk-like to relaxed (101) surface. (c) Partial charge densities computed at the valence band maximum and conduction band minimum for the $n=3$ RPP in bulk and relaxed surface. (d) Difference between the barycenter of electron and hole wavefunctions. (e) Schematics of the surface-induced exciton dissociation in RPPs with $n \geqslant 3$.

to a bandgap blueshift, whereas sub-surface lattice compression with significant octahedral distortions results in a redshift of the bandgap due to appearance of in-gap electronic states.

In order to understand the microscopic impact of the structural changes at the surface on each type of charges, localized density of states (LDOS) have been integrated around the valence band maximum (hole) and conduction band minimum (electron), leading to partial charge densities (Fig. 4c, Text V and Fig. S9a,b, SI). For all n-values, surface relaxation leads to hole wavefunctions repelled away from the surface to the bulk (Fig. 4c, bottom 
panel). A similar behaviour is observed for electrons for $n=1,2$. In sharp contrast, for $\mathrm{n}>2$, the electron gets localized mainly at the top (101) surface slab (Fig. 4c, top panel). Concomitantly, the preferential direction of electronic coupling switches from (010) to (101). From the barycenters of electron $\left(z_{e}\right)$ and hole $\left(z_{h}\right)$ partial charge density profiles (Fig. S9c,d, SI), we inspect separation of carriers $\left(\Delta z_{e} / h=z_{e}-z_{h}\right)$ and demonstrate that upon appearance of in-gap states, the electron and hole get separated (Fig. 4d). The effect is maximum for $\mathrm{n}=3, \Delta \mathrm{z}_{\mathrm{e} / \mathrm{h}}=13.2 \AA$ ( $5.5 \AA$ for $\left.\mathrm{n}=4\right)$. Its impact on optical activity is estimated by computing Kane energies ${ }^{25}$ for bulk-like and relaxed slabs (Text VI, SI). They reflect oscillator strengths of the optical-transitions and show a systematic reduction by $50 \%, 85 \%, 30 \%$ and $95 \%$ for the 4 lowest excitations of $n=3$ RPP (Table S3, SI). Such electron-hole separation at the surface is consistent with the longer photoluminescence lifetime of low-energy states reported recently. ${ }^{15}$ To account for the excitonic properties of $(\mathrm{BA})_{2}(\mathrm{MA})_{\mathrm{n}-1} \mathrm{~Pb}_{\mathrm{n}} \mathrm{I}_{3 \mathrm{n}+1}$, we further involve the semi-empirical resolution of the Bethe-Salpeter equations including quantum and dielectric confinement as well as the overlap of electron and hole wavefunctions (Text IV, SI). The latter is essential for achieving quantitative agreement between the computed exciton binding energy and the low temperature spectroscopy results. ${ }^{24}$ This can be intuited from the simplified screened electron-hole interaction (without dielectric confinement):

$$
V_{s}\left(q_{\|}\right)=\frac{-e^{2}}{2 \epsilon_{w} q_{\|}} \iint \rho_{e}\left(z_{e}\right) \rho_{h}\left(z_{h}\right) e^{-q_{\|}\left|z_{e}-z_{h}\right|} d z_{e} d z_{h}
$$

which shows that the separation of electrons and holes depicted in Fig. 4c and Fig. 4d will cause the collapse of the exciton binding energy (see Text VII and Fig. S11, SI). Fig. 4e summarizes our understanding of the formation of these low-energy states (LES) in BAbased RPPs with $n>2$, which primarily stems from surface relaxation that strongly localizes the electron at the surface and facilitates dissociation of the strongly bound bulk exciton.

LES result from the release of the strain-induced elastic energy at the L1/L2 interface (Fig. 2). From our elastic model, the amount of energy accumulated in the materials is 

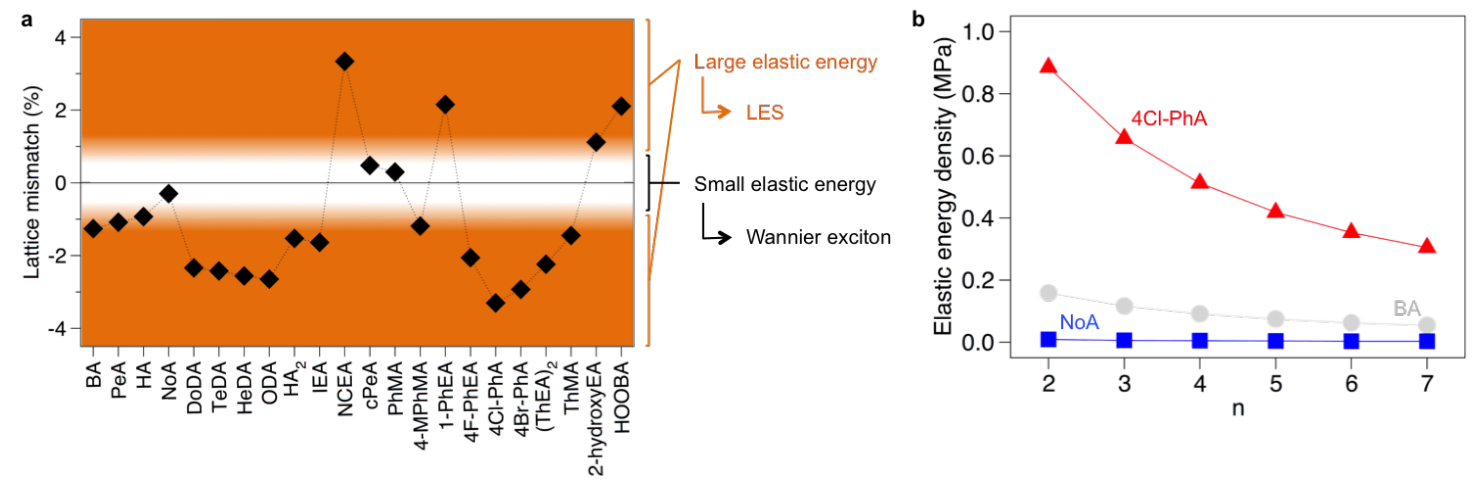

Figure 5: Design of LHPs for photovoltaics and optoelectronics. (a) Lattice mismatch between various monolayered $\mathrm{A}_{2}{ }_{2} \mathrm{PbI}_{4}$ perovskites $(\mathrm{n}=1)$ and $\mathrm{MAPbI}_{3}(I 4 \mathrm{~cm} ; \mathrm{n}=\infty)$. All data are taken from $\mathrm{X}$-ray structures resolved at room-temperature. Names for organic compounds and corresponding references are given Table S4 (SI). (b) Computed elastic energy density for heterostructures built with $\mathrm{MAPbI}_{3}$ and $(\mathrm{BA})_{2} \mathrm{PbI}_{4}$ (grey line), $\left(\mathrm{C}_{9} \mathrm{H}_{19} \mathrm{NH}_{3}\right)_{2} \mathrm{PbI}_{4}$ (NoA, blue line), and (4Cl$\left.\mathrm{C}_{6} \mathrm{H}_{4} \mathrm{NH}_{3}\right)_{2} \mathrm{PbI}_{4}$ (4Cl-PhA, red line).

directly dependent on the amplitude of lattice mismatch between layers in the heterostructure L1/L2 and as a result, tuning the LHP structure and composition can lead to drastic changes of surface properties. Using this general approach, the internal elastic energy density accumulated in the bulk of LHPs can be estimated for any composition and perovskite layer thickness. From a practical perspective, understanding the relaxation of the stored elastic energy at the surface of the LHP materials is of paramount importance and presents a perfect platform for the systematic and comprehensive evaluation and screening of LHP compounds with defined functionalities for novel devices. This concept is illustrated by changing organic cation $A^{\prime}$ in RPPs (Fig. 5a). For example, replacing BA with $\mathrm{C}_{9} \mathrm{H}_{19} \mathrm{NH}_{3}$ (NoA), which has a significantly smaller lattice mismatch, ${ }^{26}$ results in the reduction of the elastic energy density of the RPP composite by more than an order of magnitude (Fig. 5b). This would prevent surface relaxation and, in turn, formation of LES, preserving the bulk Wannier exciton. By contrast, RPPs based on an organic cation inducing a larger mismatch, namely $\left(4 \mathrm{Cl}-\mathrm{C}_{6} \mathrm{H}_{4} \mathrm{NH}_{3}\right)_{2} \mathrm{PbI}_{4}$ (4Cl-PhA), ${ }^{27}$ undergoes increased strain (Fig. 5a), thus larger elastic energy density (Fig. 5b) that should favour significant (101) surface relaxation suitable for e-h carrier separation.

In summary, surface relaxation effects in layered hybrid perovskite materials have been 
thoroughly investigated. We introduced the first generic elastic model for LHPs accounting for the internal elastic energy accumulated in the material bulk as a result of lattice mismatch. We show that the mechanical energy is more efficiently relaxed in LHPs thanks to the additional rotational degrees of freedom provided by the perovskite octahedra-based framework. This is further demonstrated using electronic structure calculations of the surface relaxation of perovskite layers in a well-documented family of RPPs, and we discovered a critical layer thickness above which the surface reorganization becomes significant. This consequently leads to the formation of lower energy electronic states rationalizing and confirming experimental observations. ${ }^{15}$ Our observation of electronic bandgap shifts and exciton dissociation at the surface, depending on the layered perovskite structure distinguishes these materials from their $3 \mathrm{D} \mathrm{APbI}_{3}(\mathrm{~A}=$ cation; $\mathrm{n}=\infty)$ counterparts and pave the way to unique tailored properties and functionalities for optoelectronic, solid-state lighting or photovoltaic applications.

\section{Methods}

DFT calculations are conducted with the SIESTA code. ${ }^{28}$ The non-local van der Waals density functional of Dion et al. corrected by Cooper (C09) is used for geometry optimizations. ${ }^{29,30} \mathrm{SOC}$ is taken into account through the on-site approximation as proposed by Fernández-Seivane et al. ${ }^{31}$ To prevent conflicts between the on-site treatment and the non-locality of $\mathrm{C} 09$, single points calculations are conducted with the revPBE functional on which C09 is based. The dipole induced in slabs is treated with the dipole correction as implemented in SIESTA. ${ }^{32}$ Core electrons are described with Troullier-Martins pseudopotentials. ${ }^{33}$ The valence wavefunction is developed over a double- $\zeta$ polarized basis set

of finite-range numerical pseudoatomic orbitals. ${ }^{34}$ An energy cutoff of 150 Ry for real-space mesh size is used. Noteworthy, DFT underestimates the bandgap closing upon surface relaxation in RPPs with $\mathrm{n}>2$. Turning to larger supercells, e.g. doubled, is likely to allow for additional distortions, which in turn shall further close the bandgap. Unfortunately, such 
size doubling is computationally too demanding. Besides, for complex spinor Bloch functions (Kane model) we used the ABINIT package ${ }^{35}$ with GGA-revPBE gradient correction for exchange-correlation, ${ }^{36}$ ABINIT projector augmented-wave (PAW) datasets ${ }^{37}$ as pseudpotentials for $\mathrm{Pb}$, I, and $\mathrm{Cs}$ and an energy cutoff of $19 \mathrm{Ha}(517 \mathrm{eV})$ for the plane-wave basis set. Primitive cells were used for the computation of the electronic band dispersions with Monkhorst-Pack grids for reciprocal space integration of: $4 \times 4 \times 1$ for $n=1$ and $2 \times 2 \times 4$ for $\mathrm{n}=2,3$ and 4 . Spin-orbit coupling was included in all calculations. As plane wave calculations are more demanding than the localized basis sets counterpart, organic cations have been substituted by Cs atoms. This substitution is known to leave the band dispersion unaffected close to the Fermi level and, in fact, for $\mathrm{n}=3$ we obtain a comparable bandgap closing $(128 \mathrm{meV})$ and similar spinorial components than with SIESTA and all atoms.

\section{Acknowledgements}

The work in France was supported by Agence Nationale pour la Recherche (TRANSHYPERO project) and was granted access to the HPC resources of [TGCC/CINES/IDRIS] under the allocation 2017-A0010907682 made by GENCI. The work at Los Alamos National Laboratory (LANL) was supported by LANL LDRD program (J-C.B., W.N., S.T., A.D.M.) and was partially performed at the Center for Nonlinear Studies. The work was conducted, in part, at the Center for Integrated Nanotechnologies (CINT), a U.S. Department of Energy, Office of Science user facility. Work at Northwestern University was supported by grant SC0012541 from the U.S. Department of Energy, Office of Science. C.C.S. and M.G.K. acknowledge the support under ONR Grant N00014-17-1-2231. 


\section{Supporting Information Available}

Additional details on the improper flexoelastic model, tilting angles description, construction of the (101) surface, strain energetics, surface band structures and partial electronic densities, Kane models, excitonic properties of RPPs, references for Fig. 5.

\section{References}

(1) Zwanenburg, F. A.; Dzurak, A. S.; Morello, A.; Simmons, M. Y.; Hollenberg, L. C. L.; Klimeck, G.; Rogge, S.; Coppersmith, S. N.; Eriksson, M. A. Rev. Mod. Phys. 2013, 85, 961.

(2) del Alamo, J. A. Nature 2011, 479, 317-323.

(3) Hwang, H. Y.; Iwasa, Y.; Kawasaki, M.; Keimer, B.; Nagaosa, N.; Tokura, Y. Nature Mater. 2012, 11, 103-113.

(4) Haruyama, J.; Sodeyama, K.; Han, L.; Tateyama, Y. Acc. Chem. Res. 2016, 49, 554-561.

(5) Murali, B.; Yengel, E.; Yang, C.; Peng, W.; Alarousu, E.; Bakr, O. M.; Mohammed, O. F. ACS Energy Lett. 2017, 2, 846-856.

(6) Saparov, B.; Mitzi, D. B. Chem. Rev. 2016, 116, 4558-4596.

(7) Pedesseau, L.; Sapori, D.; Traore, B.; Robles, R.; Fang, H.-H.; Loi, M. A.; Tsai, H.; Nie, W.; Blancon, J.-C.; Neukirch, A. J.; Tretiak, S.; Mohite, A. D.; Katan, C.; Even, J.; Kepenekian, M. ACS Nano 2016, 10, 9776-9786.

(8) Li, W.; Wang, Z.; Deschler, F.; Gao, S.; Friend, R. H.; Cheetham, A. K. Nat. Rev. Mater. 2017, 2, 16099.

(9) Tsai, H.; Nie, W.; Blancon, J.-C.; Stoumpos, C. C.; Asadpour, R.; Harutyunyan, B.; Neukirch, A. J.; Verduzco, R.; Crochet, J. J.; Tretiak, S. et al. Nature 2016, 536, 312-317.

(10) Mitzi, D. B.; Feild, C. A.; Harrison, W. T. A.; Guloy, A. M. Nature 1994, 369, 467.

(11) Kagan, C. R.; Mitzi, D. B.; Dimitrakopoulos, C. D. Science 1999, 286, 945. 
(12) Yuan, M.; Quan, L. N.; Comin, R.; Walters, G.; Sabatini, R.; Voznyy, O.; Hoogland, S.; Zhao, Y.; Beauregard, E. M.; Kanjanaboos, P.; Lu, Z.; Kim, D. H.; Sargent, E. H. Nature Nanotech. 2016, $11,872-877$.

(13) Wang, N.; Cheng, L.; Ge, R.; Zhang, S.; Miao, Y.; Zou, W.; Yi, C.; Sun, Y.; Cao, Y.; Yang, R. et al. Nature Photon. 2016, 10, 699-704.

(14) Odenthal, P.; Talmadge, W.; Gundlach, N.; Wang, R.; Zhang, C.; Sun, D.; Yu, Z.-G.; Vardeny, Z. V.; Li, Y. S. Nature Phys. 2017, 13, 894-899.

(15) Blancon, J.-C.; Tsai, H.; Nie, W.; Stoumpos, C. C.; Pedesseau, L.; Katan, C.; Kepenekian, M.; Soe, C. M. M.; Appavoo, K.; Sfeir, M. Y. et al. Science 2017, 355, 1288-1292.

(16) Even, J.; Pedesseau, L.; Katan, C. ChemPhysChem 2014, 15, 3733.

(17) Sapori, D.; Kepenekian, M.; Pedesseau, L.; Katan, C.; Even, J. Nanoscale 2016, 8, 6369.

(18) Vurgaftman, I.; Meyer, J. R. J. Appl. Phys. 2001, 89, 5815.

(19) Bimberg, D.; Grundmann, M.; Ledentsov, N. N. Quantum Dot Heterostructures; John-Wiley and Sons: 1999.

(20) Ruddlesden, S. N.; Popper, P. Acta Cryst. 1957, 10, 538-539.

(21) Ruddlesden, S. N.; Popper, P. Acta Cryst. 1958, 11, 54-55.

(22) Stoumpos, C. C.; Cao, D. H.; Clark, D. J.; Young, J.; Rondinelli, J. M.; Jang, J. I.; Hupp, J. T.; Kanatzidis, M. G. Chem. Mater. 2016, 28, 2852-2867.

(23) Davies, J. H.; Bruls, D. M.; Vugs, J. W. A. M.; Koenraad, P. M. J. Appl. Phys. 2002, 91, 4171-4176.

(24) Blancon, J.-C.; Stier, A. V.; Tsai, H.; Nie, W.; Stoumpos, C. C.; Traore, B.; Pedesseau, L.; Kepenekian, M.; Tretiak, S.; Crooker, S. A. et al. Nat. Commun. 2018, 9, 2254.

(25) Kane, E. O. Semiconductors and Semimetals; Academic Press: 1966.

(26) Lemmerer, A.; Billing, D. G. Dalton Trans. 2012, 41, 1146-1157.

(27) Liu, Z.; Yu, W.-T.; Tao, X.-T.; Jiang, M.-H.; Yang, J.-X.; Wang, L. Z. Kristallogr. NCS 2004, 219, 457-458. 
(28) Soler, J. M.; Artacho, E.; Gale, J. D.; García, A.; Junquera, J.; Ordejón, P.; Sánchez-Portal, D. J. Phys.: Condens. Matter 2002, 14, 2745-2779.

(29) Dion, M.; Rydberg, H.; Schröder, E.; Langreth, D. C.; Lundqvist, B. I. Phys. Rev. Lett. 2004, 92, 246401.

(30) Cooper, V. R. Phys. Rev. B 2010, 81, 161104(R).

(31) Fernández-Seivane, L.; Oliveira, M. A.; Sanvito, S.; Ferrer, J. J. Phys.: Condens. Matter 2006, 18, 7999.

(32) Bengtsson, L. Phys. Rev. B 1999, 59, 12301.

(33) Troullier, N.; Martins, J. L. Phys. Rev. B 1991, 43, 1993-2006.

(34) Artacho, E.; Sánchez-Portal, D.; Ordejón, P.; García, A.; Soler, J. M. Phys. Status Solidi B 1999, $215,809-817$.

(35) Gonze, X.; Amadon, B.; Anglade, P.-M.; Beuken, J.-M.; Bottin, F.; Boulanger, P.; Bruneval, F.; Caliste, D.; Caracas, R.; Côté, M. et al. Comput. Phys. Commun. 2009, 180, 2582-2615.

(36) Zhang, Y.; Yang, W. Phys. Rev. Lett. 1998, 80, 890.

(37) Torrent, M.; Jollet, F.; Bottin, F.; Zérah, G.; Gonze, X. Comput. Mater. Sci. 2008, 42, 337-351. 